\title{
Preferencias de los y las estudiantes universitarias sobre el empleo desde una perspectiva de género
}

\section{The university student preferences on employment from a gender perspective}

Amparo PÉREZ-CARBONELL y Genoveva RAMOS SANTANA

Universidad de Valencia

Recibido: Abril 2014

Aceptado: Mayo 2014

\section{Resumen}

Se presenta un estudio de encuesta sobre las preferencias que tiene el alumnado universitario al decidirse por un trabajo, lo que creen que les debe aportar y cómo debe ser la empresa en la que desearían trabajar en función de la variable género. Diferentes investigaciones, señalan que las personas desarrollan valores, expectativas y habilidades profesionales diferentes asociadas a la carrera y a la profesión en función del género. El objetivo de este trabajo es analizar los factores que llevan a los y las jóvenes a percibir el trabajo o profesión y determinar si dicha percepción es diferente por ser hombre o mujer. A través de un cuestionario ad hoc compuesto por 52 ítems de escala Likert se recoge la información a 1295 estudiantes universitarios seleccionados a través de un muestreo no probabilístico por accesibilidad y que realizan sus estudios en 28 universidades y 53 facultades españolas públicas y privadas durante el curso académico 201011. Las respuestas aportadas en dicho cuestionario son analizadas con la prueba $\mathrm{U}$ de MannWhitney y el Análisis factorial de componentes principales exploratorio con rotación varimax diferenciado por sexo. Los resultados obtenidos constatan que el alumnado universitario, desarrolla sus preferencias laborales sesgadas por estereotipos de género, aunque también se observan pequeños cambios de tendencias en dichas preferencias como, la valoración de la conciliación familiar ante la decisión de un empleo por parte de los universitarios o la búsqueda de trabajos que les lleven a retos constantes en el caso de las universitarias.

Palabras clave: estudiante universitario, estereotipo, sexo, empleo, división del trabajo.

\begin{abstract}
A survey study about the preferences held by undergraduate students to decide on a job, what they think they should provide and how the company should be as well as where they wish to work in terms of the gender variable is presented. Different studies point out that people develop values, expectations and different professional skills associated with career and profession depending on the gender. The aim of this paper is to analyze the factors that lead young people to receive the job or profession and determine whether that perception is different being male or female. Through an ad hoc questionnaire with 52 Likert scale items and a sample of 1295 college students selected through a non-probability sampling accessibility. The sample belonged to 28 universities and 53 public and private Spanish schools where the students
\end{abstract}


attended and was collected during the academic year 2010-11. The answers given in the questionnaire were analyzed with the U Mann-Whitney test and factorial exploratory principal components analysis with varimax rotation differentiated by gender. The results find that university students develop their work preferences based in gender stereotypes. Even small changes in trends are also seen in these preferences, such as the valuation of family conciliation before the employment decision or seeking jobs with constant challenges in the case of university female students.

Keywords: university students, stereotypes, sex, employmen, gender division of labour.

Estudios realizados desde hace ya algunos años (Inglehart, 1998) permiten constatar que se ha dado un cambio en lo que nos motiva a las personas a trabajar, es decir, la importancia por los ingresos y la seguridad va dejando paso a la búsqueda de un trabajo interesante y significativo, en el que se requiere una mayor autonomía y autorrealización personal.

Desde este marco, somos conscientes que la inserción laboral de los y las jóvenes depende en buena medida de su conducta hacia el mercado de trabajo. Sin embargo, esa conducta recibe la influencia de numerosos factores como los valores, las creencias, las motivaciones, las preferencias laborales así como el grado de iniciativa y el género, entre otros.

El género, como ya se destaca en anteriores trabajos universitarios (Elejabeita, 1995; Navarro y Casero, 2012 y Olivares y Olivares, 2013), es un factor diferenciador en las elecciones de estudios y trabajos de los y las jóvenes. Las revisiones de distintas investigaciones teórico-empíricas muestran que existe justificación suficiente para creer que desarrollan valores, expectativas y habilidades profesionales asociadas a la carrera y a la profesión diferentes en función del género (Nash y Marre, 2003; Blosch, 2005; Sebastián et al., 2005, Castillo y Rodríguez Sedano, 2011; Sánchez et al., 2011; Pérez-Carbonell y Ramos, 2013). De forma específica, en estos estudios se señala que la identidad de género estereotipada y adquirida desde la infancia estimula a los varones y desalienta a las mujeres a encaminarse hacia actividades propias de trabajos científicos y técnicos. Así, uno de los motivos que explica la poca presencia de mujeres en las carreras profesionales científicas y tecnológicas podría deberse a que dichas carreras están pensadas desde la hegemonía de los valores masculinos (Rosser, 1998; Falkner, 2000; Wajcman, 2000; Kulis, Sicotte y Collins, 2002). De ahí que esta segregación por género de las carreras universitarias se relacione con la división sexual del trabajo otorgando diferente valoración al rol social de varones y mujeres y reflejando, a su vez, una segregación horizontal y vertical del mercado de trabajo (Olivares y Olivares, 2013). Es decir, aunque las profesiones retributivas no son, como en épocas anteriores, un reducto masculinizado (Martin y Miguel, 1982), en el mercado de trabajo se sigue percibiendo la dimensión de género articulada en distinción de actividades masculinas y femeninas. Sin embargo, y como bien señalan, Iglesias y Llorente (2010), aunque esta segregación laboral es un fenómeno estructural y persistente del mercado de trabajo, no debemos percibirla como una realidad neutra o invariable, hecho que suele ocurrir entre los y las estudiantes universitarias. 
De igual manera que a Gómez Bueno (2001) y a Olivares y Olivares (2013), nos preocupa esta pérdida de enfoque crítico en las generaciones jóvenes al asumirse el discurso sociopolítico de superación de la desigualdad del mercado laboral.

La elección del itinerario profesional y del ámbito laboral están determinados por factores internos, como las aptitudes, los intereses y las motivaciones; por factores externos como la familia, el entorno social, cultural y económico (Barbera, Ramos y Candela, 2006), además de diferenciado por el género. Así podemos hallar, por ejemplo, que las mujeres prefieran las actividades relacionadas con la enseñanza y con el rol tradicional femenino de cuidado y atención a los demás; mientras que los hombres remarquen en la elección de profesiones el prestigio social y económico (Silván-Ferrero et al., 2005). Concretamente, en el ámbito profesional, los estereotipos de género vinculan la masculinidad con características como la competitividad, la independencia, la fuerza o la orientación a resultados. Mientras, la feminidad se vincula con la colaboración, el cuidado de los demás, las relaciones interpersonales, la demostración de las emociones o la sensibilidad (DeArmond et al., 2006). A su vez, algunas investigaciones (Padilla, 2002; Patton y McMahon, 2006; Sánchez et. al., 2011) revelan que las mujeres escogen carreras profesionales más infra-desarrolladas que los varones al estar afectadas no solo por los factores externos (socio-laborales) sino también por las propias autolimitaciones y factores psicológicos. Autores como Suárez (2004) y Navarro y Sallé (2007), señalan la existencia de barreras que dificultan el desarrollo profesional de la mujer, como pueden ser: por un lado, los valores que éstas y éstos hacen del trabajo, las oportunidades de promoción, los estilos de liderazgo femenino, los nuevos estereotipos sobre las mujeres directivas, etc.. $\mathrm{y}$, por otro lado, los procesos que conducen a la toma de decisiones vocacionales estereotipadas en función del género, como pueden ser la autoeficacia, la autoconfianza, los fenómenos de autoexclusión, la motivación y las expectativas laborales entre otros (Sánchez et al., 2011). Algunas investigaciones que han analizado a la población femenina potencialmente emprendedora, han puesto de manifiesto que el nivel de deseo de crear una empresa propia es mayor en hombres que en mujeres (González, 2001; Rodríguez y Santos, 2008). En esta línea, Ventura y Quero (2013) destacan que el incremento de la tasa de emprendimiento en el sector femenino requiere actuar sobre la valoración de los resultados y el contexto de seguridad que es percibido por la existencia de asesoramiento y ayuda a la mujer emprendedora, lo que no sucede en los hombres. Así, el origen de algunas de estas diferencias se encuentra en factores extrínsecos e intrínsecos a la mujer que resultan complementarios: menor propensión al riesgo, un perfil menos innovador de los comportamientos y menor necesidad de logro e independencia, entre otros (Forson y Özbilgin, 2003).

Destacar, que estudios recientes siguen evidenciando que las responsabilidades familiares afectan más a la mujer que al hombre en la elección profesional y en el desarrollo laboral. Las dificultades en este sentido, evidencian los problemas de conciliación entre la vida personal, familiar y laboral, provocando que algunas mujeres tengan que decidir y elegir entre la vida familiar y la carrera profesional o el mercado laboral (Pons et al., 2013). 
Dentro de este marco, somos conscientes que las preferencias ante el trabajo en los y las jóvenes universitarias dependen en buena medida -sin atender para nada a la situación económica del momento- de sus actitudes y conductas hacia lo que buscan y esperan del mercado de trabajo. Actitudes hacia el trabajo, que reciben la influencia de numerosos factores personales que conforman su manera de ser, como son: los valores, las preferencias laborales, el grado de iniciativa, las creencias, las motivaciones, el estatus, el nivel de estudios y el género. Las creencias sociales sobre el género, configuran el modelo de organización social impregnando tradiciones, costumbres y relaciones de las personas, lo cual influye directamente en nuestras creencias, actitudes y expectativas laborales.

Actualmente, en la socialización del rol de género que han de desempeñar los hombres y las mujeres se siguen cometiendo errores que llevan a perpetuar formas de hacer y actuar contrarias a los nuevos conceptos como puede ser el de nuevas masculinidades. Por ejemplo, en lo referente a la implicación paternal y concretamente en las relaciones entre el trabajo y la paternidad, intervienen factores ligados al trabajo en sí. Esto es, hay trabajos que en realidad terminan siendo obstáculos o activadores potencialmente significativos de dicha implicación paternal (Russell y Hwang, 2004).

\section{Objetivos del estudio}

Desde este marco contextual, nos planteamos como objetivo principal de este estudio comprobar, si las y los jóvenes universitarios, presentan respecto a la empresa en la que desearían trabajar, percepciones diferentes. Es decir, percepciones estereotipadas o no en función del género.

Concretamente se investiga sobre:

- Las diferentes percepciones/preferencias que el alumnado universitario tiene cuando busca un trabajo, respecto a lo qué piensa que le debe aportar dicho trabajo; y respecto al tipo de empresa en la que les gustaría trabajar cuando finalicen su formación universitaria.

- Los factores o constructos que subyacen en dichas percepciones/preferencias y que guiarán las decisiones que ante el ámbito laboral asuman.

\section{Método}

\section{Participantes}

Los participantes de este estudio son 1295 estudiantes, 556 varones $(45.60 \%)$ y 664 mujeres (54.40\%). Todos y todas, universitarios/as españoles/as con una media de edad de 21 años, no insertados/as en el mundo laboral, pertenecientes a 28 Universidades y 53 Facultades tanto públicas como privadas durante el curso académico 2010-2011. La mitad de los casos - un 61.5\%- es alumnado que pertenece a la Rama de conocimiento de Ciencias Sociales y Jurídicas -ver tabla 1-. La selección de las personas que responden, se realiza por muestreo no probabilístico de cuotas o accidental. Las 
condiciones para cumplimentar el cuestionario se establecieron en: ser universitario o universitaria de nacionalidad española, tener entre 18 y 25 años y no haber realizado ningún trabajo remunerado.

\begin{tabular}{lrccc}
\hline \multicolumn{1}{c}{ RAMA } & N & \% Total & \% Hombres & \% Mujeres \\
\hline Artes y Humanidades & 129 & $10.60 \%$ & $5.6 \%$ & $5.0 \%$ \\
Ciencias & 41 & $3.40 \%$ & $1.6 \%$ & $1.7 \%$ \\
Ciencias de la Salud & 163 & $13.30 \%$ & $6.5 \%$ & $6.8 \%$ \\
Ciencias Sociales y Jurídicas & 751 & $61.50 \%$ & $22.1 \%$ & $39.3 \%$ \\
Ingeniería y Arquitectura & 138 & $11.30 \%$ & $9.8 \%$ & $1.6 \%$ \\
\multicolumn{1}{c}{ Total } & 1222 & $100.00 \%$ & $45.6 \%$ & $54.4 \%$ \\
& & & $\mathrm{~N}=556$ & $\mathrm{~N}=664$ \\
\hline
\end{tabular}

Tabla 1. Porcentaje de sujetos en el estudio por rama de conocimiento y por sexo

Esta descripción de los y las participantes nos permite señalar -al igual que lo hacen otros estudios como el ya presentado de Aguado (2008)-, que cuando nos referirnos a los estudiantes universitarios en general, no lo hacemos asumiéndolos como un grupo social homogéneo, sino siendo conscientes que son estudiantes universitarios no iguales en su condición académica, social, económica, cultural. Por tanto, del grupo con el que trabajamos debemos decir que es un grupo social heterogéneo que comparte una identidad ante una situación concreta: el contexto universitario en el que se encuentra en este momento de su vida.

\section{Instrumentación}

La investigación se ha desarrollado aplicando un método tipo survey. Para ello, se diseña y aplica un cuestionario ad hoc conformado, tras la revisión de un grupo de expertos/as, por 52 ítems extraídos de dos estudios considerados de gran interés social: El estudio realizado por la Fundación Universidad - Empresa denominado “Compromiso en la Empresa: Compromiso Laboral de los universitarios 2008/2009" y la encuesta de Metroscopia llevada a cabo en España bajo la supervisión de Javier Elzo y denominada "European Values Study Master Questionnaire 2008”.

Atendiendo a la validez de contenido, señalar que los ítems fueron seleccionados de los citados estudios aunque se realizaron modificaciones en su formulación que permitieron su adaptación a las características específicas de las audiencias de este estudio. La escala utilizada para la valoración de los ítems es tipo Likert de siete puntos, en la que 1 indica que, para quien responde, lo propuesto es "nada importante" y 7 "muy importante". Es decir, los valores altos de la escala coinciden con una mayor percepción o una más alta preferencia hacia el objeto de estudio por parte de los/las estudiantes y los valores bajos indican una menor percepción o menor preferencia hacia los reactivos planteados. Todos los ítems se establecen bajo tres formulaciones que se han planteado expresamente para este cuestionario -ver tabla 2- : 
- A la hora de buscar o decidirme por un trabajo valoro.... Aquí se presentan diferentes aspectos sobre tópicos en la preferencia o elección de los trabajos.

- Pienso que un/el trabajo me debe aportar.... Apartado que tiene como finalidad averiguar qué aspectos desean las y los jóvenes que les aporte el trabajo.

- Cuando finalice mi formación universitaria me gustaría trabajar en una empresa que.... Busca conocer cuál es el ideal de empresa donde desearían trabajar las universitarias y los universitarios.

El instrumento diseñado se sometió a reflexión y comprobación sobre su calidad como instrumento de recogida de información y en tal proceso se obtuvo un alto nivel de consistencia interna global $(\alpha=0.95)$. Así pues, los ítems presentados son adecuados para obtener información sobre la percepción que las y los universitarios tienen sobre su futuro trabajo y/o empresa.

\section{Análisis}

Los análisis, que se han realizado mediante el paquete estadístico SPSS para Windows, versión 20.0, se han dirigido a dar respuesta a los objetivos del estudio. Concretamente se plantean los siguientes:

- Análisis no paramétrico para trabajar las diferencias entre las percepciones, en concreto la prueba U de Mann-Whitney, como alternativa a la prueba $t$ sobre diferencias de medias al no cumplirse -en los datos recogidos- los supuestos de normalidad y homoscedasticidad.

- Análisis factorial de componentes principales exploratorio con rotación varimax diferenciado por sexo, con el objetivo de determinar cuáles son "los factores o constructos" que subyacen en las decisiones que ante el ámbito laboral asumen ambos grupos. Análisis, que ha permitido comentar las preferencias que señalan las universitarias y los universitarios ante su futuro ingreso en el mundo laboral.

\section{Resultados}

La mayoría del alumnado encuestado responde al total de los 52 ítems que componen el cuestionario y además, lo hacen con una tendencia generalizada de valoraciones elevadas en las respuestas. De hecho, un $78.85 \%$ de los ítems planteados tienen una valoración $\geq 5$ puntos en una escala de 1 a 7 .

En el análisis de las respuestas se observa que las universitarias y los universitarios, al decidirse por un trabajo no valoran en exceso, que la empresa sea prestigiosa así como el hecho de poder llegar a crear su propia empresa. Sin embargo, sí que buscan trabajos en los que se sientan bien tratados/as, donde se consiga un buen ambiente laboral, que les resulte interesante/gratificante y que les permita la conciliación con su vida familiar. También valoran la satisfacción y la estabilidad o seguridad, con 
intención de poder desarrollar toda su vida profesional en la misma empresa, aun siendo relevante la buena remuneración económica. De los 52 ítems del cuestionario aparecen diferencias significativas en 34 ítems. Así pues, hay diferencias en un 65.38\% de los ítems a los que responden los y las jóvenes universitarios/as.

En los primeros 20 ítems se plantea el grado de importancia que los y las estudiantes dan a los aspectos que recogen información sobre lo que ellos y ellas valoran a la hora de buscar o decidirse por un trabajo -tabla 2-. Como se aprecia en la tabla aparecen diferencias en la respuesta dada entre ambos grupos, lo que permite afirmar que al decidirse por un trabajo hay aspectos que las universitarias y los universitarios valoran de forma diferente -ver tabla 2-. Existen diferencias al valorar: cómo se sienten ante el trato que reciben (ítem 2); el ambiente de trabajo en el que estarían (ítem 3); el nivel de seguridad (ítem 4) y de gratificación que les ha de ofrecer la empresa (ítem 8); la posibilidad de seguir su formación tanto a nivel profesional (ítem 9) como para las propias necesidades de la empresa (ítem 10); el nivel de utilidad que tiene la empresa para con la sociedad (ítem 12); que nos les haga sentir presión (ítem 13); que les permita tener iniciativas (ítem 14); tratar con personas (ítem 15) y que lo que tengan que hacer en su puesto de trabajo se ajustase a las competencias que tienen como profesionales (ítem 17). Diferencias, que reflejan que son las universitarias las que valoran todos estos aspectos como más importantes. Mientras que en las diferencias que se manifiestan en la cuestión de tener buenos ingresos (ítem 1); amplías vacaciones (ítem6) y en la de poder llegar a crear una empresa propia (ítem 20) son los universitarios los que señalan que son aspectos de mayor relevancia. Por ramas de conocimiento y atendiendo a los 14 ítems en los que se dan las diferencias, el alumnado de Ciencias Sociales y Jurídicas, da una respuesta coincidente con la ya comentada; en Ciencias de la Salud las universitarias reflejan mayor acuerdo que sus compañeros con la idea de poder crear una empresa propia (ítem20); en Ciencias siempre son las universitarias las que presentan mayor valoración a todos estos ítems a excepción del ítem 13 y 20, en los que es al contrario; en Artes y Humanidades se da un cambio, pues los universitarios valoran como más importantes además de los ítems 6 y 20, el 12, el 13 y el 14, siendo en Ingeniería y Arquitectura dónde las universitarias valoran como más importante los ítems $4,9,10,12$ y 14 , mientras que sus compañeros lo hacen en todos los demás ítems. 


\begin{tabular}{|c|c|c|c|c|c|c|}
\hline \multirow[b]{3}{*}{ Item } & \multicolumn{6}{|c|}{ A la hora de buscar o decidirme por un trabajo valoro... } \\
\hline & & \multicolumn{2}{|c|}{ Chicas } & \multicolumn{2}{|c|}{ Chicos } & \multirow{2}{*}{ Diferencias } \\
\hline & & Md & $\boldsymbol{X}$ & Md & $\boldsymbol{X}$ & \\
\hline 1 & Tener buenos ingresos. & 6 & 5.51 & 6 & 5.64 & $(*)$ \\
\hline 2 & Sentirme bien tratado/a. & 7 & 6.37 & 6 & 6.13 & $(* *)$ \\
\hline 3 & Tener un buen ambiente de trabajo. & 6 & 6.25 & 6 & 6.06 & $(* *)$ \\
\hline 4 & Que me ofrezca seguridad. & 6 & 5.83 & 6 & 5.47 & $(* *)$ \\
\hline 5 & Tener horario flexible. & 5 & 5.27 & 5 & 5.23 & \\
\hline 6 & Tener amplías vacaciones. & 5 & 4.75 & 5 & 5.05 & $(* *)$ \\
\hline 7 & $\begin{array}{l}\text { Tener posibilidades de promocionar en la } \\
\text { empresa. }\end{array}$ & 5 & 5.15 & 5 & 5.16 & \\
\hline 8 & Que sea un trabajo interesante/ gratificante. & 6 & 6.16 & 6 & 5.90 & $(* *)$ \\
\hline 9 & $\begin{array}{l}\text { Que me permita seguir formándome en mi } \\
\text { profesión. }\end{array}$ & 6 & 5.92 & 6 & 5.56 & $(* *)$ \\
\hline 10 & $\begin{array}{l}\text { Que me permita seguir formándome para las } \\
\text { necesidades de la empresa. }\end{array}$ & 5 & 5.19 & 5 & 4.81 & $(* *)$ \\
\hline 11 & $\begin{array}{l}\text { Que me permita conciliar el trabajo con mi vida } \\
\text { familiar. }\end{array}$ & 6 & 6.07 & 6 & 5.97 & \\
\hline 12 & Que sea útil para la sociedad. & 6 & 5.40 & 5 & 4.89 & $(* *)$ \\
\hline 13 & Que no me haga sentir agobio o presión. & 6 & 5.56 & 6 & 5.35 & $(*)$ \\
\hline 14 & Que me permita tener iniciativas. & 6 & 5.59 & 6 & 5.43 & $(*)$ \\
\hline 15 & Que me permita tratar con gente. & 6 & 5.56 & 5 & 5.13 & $(* *)$ \\
\hline 16 & Que tenga que asumir responsabilidades. & 5 & 5.06 & 5 & 4.95 & \\
\hline 17 & $\begin{array}{l}\text { Que se ajuste a las capacidades/habilidades/ } \\
\text { competencias que tengo. }\end{array}$ & 6 & 5.82 & 6 & 5.65 & $(* *)$ \\
\hline 18 & $\begin{array}{l}\text { Que me permita tener autonomía en la toma de } \\
\text { decisiones. }\end{array}$ & 6 & 5.46 & 6 & 5.41 & \\
\hline 19 & Que sea una empresa prestigiosa. & 4 & 3.85 & 4 & 3.85 & \\
\hline 20 & Poder llegar a crear mi propia empresa. & 4 & 3.89 & 4 & 4.15 & $(*)$ \\
\hline
\end{tabular}

Md: Mediana ítem; $X$ : Media ítem

(*): Nivel de significación menor .05; (**): Nivel de significación menor .01

Tabla 2. Estadísticos y diferencias ítem (Bloque A la hora de buscar o decidirme por un trabajo valoro...)

De los 19 ítems que pretenden conocer qué es lo que universitarios y universitarias piensan que un trabajo les debe aportar, se encuentran diferencias significativas en 13 de ellos -ver tabla 3-. Las universitarias, al valorar lo que un trabajo les debe aportar, dan mayor importancia al aprendizaje constante (ítem 21); al sentimiento de utilidad (ítem 22); a las nuevas experiencias (ítem 23); a las buenas relaciones con otras personas (ítem 24); a la integración en un equipo de trabajo (ítem 25); a innovar y/o experimentar (ítem 28); a sentirse aceptada por sus compañeros/as (ítem 29); dar servicio a otras personas (ítem 30); a la satisfacción por el trabajo que realizan (ítem 31); a la independencia en el día a día (ítem 32); a la capacidad de orientar a otros/as (ítem 33) y a la admiración y reconocimiento, por parte de compañeros/as y/o jefes 
(ítem 35). Sin embargo, la tendencia se invierte en la valoración sobre el tema económico, donde los universitarios son los que ofrecen valoraciones más altas ante buena remuneración aunque el prestigio de la empresa sea menor (ítem 38).

Por Ramas de conocimiento, las diferencias entre ellas y ellos son muy similares a las ya comentadas de forma global, a excepción de nuevo del grupo de alumnado de Ingeniería y Arquitectura, donde los universitarios valoran como más importante que sus compañeras, no solo el aspecto económico (ítem 38) sino también los ítems 21, 23, $24,29,30,32$ y 33 .

\begin{tabular}{|c|c|c|c|c|c|c|}
\hline \multicolumn{7}{|c|}{ Pienso que un/el trabajo me debe aportar... } \\
\hline \multirow[b]{2}{*}{ Item } & & \multicolumn{2}{|c|}{ Chicas } & \multicolumn{2}{|c|}{ Chicos } & \multirow{2}{*}{ Diferencias } \\
\hline & & Md & $\boldsymbol{X}$ & Md & $\boldsymbol{X}$ & \\
\hline 21 & Aprendizajes constantemente. & 6 & 5.57 & 5 & 5.18 & $(* *)$ \\
\hline 22 & Sentimiento de utilidad. & 6 & 6.00 & 6 & 5.66 & $(* *)$ \\
\hline 23 & Nuevas experiencias. & 6 & 5.78 & 6 & 5.46 & $(* *)$ \\
\hline 24 & Buenas relaciones con otras personas. & 6 & 5.94 & 6 & 5.66 & $(* *)$ \\
\hline 25 & $\begin{array}{l}\text { Posibilidad de integración en un equipo de } \\
\text { trabajo. }\end{array}$ & 6 & 5.73 & 5 & 5.29 & $(* *)$ \\
\hline 26 & Retos constantes. & 5 & 5.21 & 5 & 5.10 & \\
\hline 27 & Éxito profesional. & 6 & 5.69 & 6 & 5.62 & \\
\hline 28 & Innovar y/o experimentar. & 6 & 5.52 & 5 & 5.29 & $(* *)$ \\
\hline 29 & $\begin{array}{l}\text { Sentimiento de aceptación entre los/as } \\
\text { compañeros/as. }\end{array}$ & 6 & 5.95 & 6 & 5.60 & $(* *)$ \\
\hline 30 & El dar servicio a otras personas. & 6 & 5.74 & 5 & 5.21 & $(* *)$ \\
\hline 31 & Satisfacción por el trabajo que realizo. & 7 & 6.36 & 6 & 6.05 & $(* *)$ \\
\hline 32 & Independencia en el día a día. & 6 & 5.56 & 5 & 5.34 & $(* *)$ \\
\hline 33 & Capacidad de orientar a otras personas. & 6 & 5.61 & 5 & 5.02 & $(* *)$ \\
\hline 34 & Prestigio profesional. & 5 & 5.20 & 5 & 5.13 & \\
\hline 35 & $\begin{array}{l}\text { Admiración y reconocimiento por parte de } \\
\text { compañeros/as } y / \text { o jefes/as. }\end{array}$ & 6 & 5.35 & 5 & 5.16 & $(*)$ \\
\hline 36 & Estabilidad laboral (trabajo fijo). & 6 & 6.10 & 7 & 6.03 & \\
\hline 37 & $\begin{array}{l}\text { Buena remuneración económica, aun a costa de } \\
\text { la satisfacción por el trabajo. }\end{array}$ & 5 & 4.92 & 5 & 5.06 & \\
\hline 38 & $\begin{array}{l}\text { Buena remuneración económica aunque el } \\
\text { prestigio sea menor. }\end{array}$ & 5 & 5.03 & 5 & 5.23 & $(* *)$ \\
\hline 39 & $\begin{array}{l}\text { Buena remuneración económica, aunque el } \\
\text { trabajo no sea fijo. }\end{array}$ & 5 & 4.55 & 5 & 4.63 & \\
\hline
\end{tabular}

Md: Mediana ítem; X: Media ítem

(*): Nivel de significación menor .05; (**): Nivel de significación menor .01

Tabla 3. Estadísticos y diferencias ítem (Bloque lo que universitarios y universitarias piensan que un trabajo les debe aportar

En los últimos 13 ítems del cuestionario, se propone analizar el ideal de empresa en la que a los y las jóvenes les gustaría trabajar al finalizar sus estudios, y vemos como 
hay ocho ítems en los que se encuentran diferencias significativas entre la valoración dada por las universitarias y los universitarios- ver tabla 4-.

Diferencias, que muestran como para las universitarias es más importante que para sus compañeros que la empresa en la que trabajen les permita seguir estudiando (ítem 41); realizarse conjuntamente con otras personas (ítem 43); ser innovadoras (ítem45); creativas (ítem 46); ejercer su profesión aunque el sueldo sea más bajo (ítem 48) y ser una empresa donde puedan desarrollar toda su vida profesional (ítem 52). Sin embargo, los universitarios responden dando mayor importancia que sus compañeras a aspectos tales como que desearían trabajar en una empresa donde tenga que dirigir a los demás en contraposición a que le dirijan (ítem 50), y una empresa que le deje mucho tiempo libre aunque el sueldo sea menor (ítem 51). En cuanto a la revisión de las diferencias por ramas, podemos decir que el alumnado de Ciencias de la Salud y el de Ciencias Sociales y Jurídicas responde igual que lo hacen las universitarias y los universitarios considerados en conjunto; mientras que en las otras tres ramas hay algunos cambios. Así, en Artes y Humanidades los alumnos valoran como más importante que sus compañeras los ítems 43, 45 y 51; en Ciencias, en todos los ítems en los que hay diferencias significativas, son las universitarias las que dan valoraciones más altas y en Ingeniería y Arquitectura solo el ítem 51 tiene una valoración más alta en los chicos que en las chicas.

\begin{tabular}{|c|c|c|c|c|c|c|}
\hline \multicolumn{7}{|c|}{ Cuando finalice mi formación universitaria me gustaría trabajar en una empresa que... } \\
\hline \multirow[b]{2}{*}{ Item } & & \multicolumn{2}{|c|}{ Chicas } & \multicolumn{2}{|c|}{ Chicos } & \multirow{2}{*}{ Diferencias } \\
\hline & & Md & $\boldsymbol{X}$ & Md & $\boldsymbol{X}$ & \\
\hline 40 & Me ofrezca un trabajo seguro (fijo). & 6 & 6.00 & 6 & 5.94 & \\
\hline 41 & Me permita seguir estudiando. & 6 & 5.35 & 5 & 5.02 & $(* *)$ \\
\hline 42 & $\begin{array}{l}\text { Donde tenga responsabilidad y por ello tenga que } \\
\text { decidir y dar cuenta de lo que hago. }\end{array}$ & 5 & 4.99 & 5 & 4.91 & \\
\hline 43 & $\begin{array}{l}\text { Donde el trabajo me permita realizarme } \\
\text { conjuntamente con otras personas. }\end{array}$ & 6 & 5.60 & 5 & 5.22 & $(* *)$ \\
\hline 44 & Me permita viajar. & 5 & 4.99 & 5 & 5.12 & \\
\hline 45 & Sea innovadora. & 6 & 5.44 & 5 & 5.08 & $(* *)$ \\
\hline 46 & Donde yo pueda ser creativo/a. & 6 & 5.57 & 6 & 5.33 & $(* *)$ \\
\hline 47 & Me mantenga en continuo cambio. & 5 & 4.85 & 5 & 4.67 & \\
\hline 48 & $\begin{array}{l}\text { Me permita ejercer mi profesión aunque el sueldo } \\
\text { sea más bajo. }\end{array}$ & 5 & 5.10 & 5 & 4.60 & $(* *)$ \\
\hline 49 & $\begin{array}{l}\text { Me proporcione una buena remuneración } \\
\text { económica aunque el prestigio sea menor. }\end{array}$ & 5 & 4.94 & 5 & 5.09 & \\
\hline 50 & $\begin{array}{l}\text { Donde tenga que dirigir a los demás en } \\
\text { contraposición a que me dirijan. }\end{array}$ & 4 & 4.15 & 5 & 4.53 & $(* *)$ \\
\hline 51 & $\begin{array}{l}\text { Me deje mucho tiempo libre aunque el sueldo sea } \\
\text { menor. }\end{array}$ & 4 & 4.29 & 5 & 4.61 & $(* *)$ \\
\hline 52 & Me permita desarrollar toda mi vida profesional. & 6 & 5.84 & 6 & 5.62 & $(* *)$ \\
\hline
\end{tabular}

Md: Mediana ítem; $X$ : Media ítem

(*): Nivel de significación menor .05; (**): Nivel de significación menor .01

Tabla 4. Estadísticos y diferencias ítem (Bloque el ideal de empresa en la que a los y las jóvenes les gustaría trabajar al finalizar sus estudios 
Para determinar los factores o constructos que subyacen en las percepciones que tienen las universitarias y los universitarios y que guían sus decisiones ante la búsqueda de empleo, se ha elegido el análisis factorial de componentes principales exploratorio con rotación varimax. Esta elección se ha realizado con el fin de encontrar una estructura factorial simple, clara y que pueda ser interpretada con facilidad. Decisión que avalamos por el hecho de que el número de alumnado con el que trabajamos en el estudio, tanto en universitarias como en universitarios, es el doble que el número de ítems así como que en ambos grupos de estudio, su $N$ supera diez veces el número de ítems (Costello y Osborne, 2003). Para conocer la estructura factorial del cuestionario, se utiliza la prueba Kaiser-Meyer-Olkin KMO and Bartlett y tanto en las universitarias como en los universitarios se obtiene un índice de adecuación muestral de .938 y de .930 , respectivamente y con $\mathrm{p} \leq 0.01$, en ambos casos. Resultados que indican que los grupos son adecuados para el análisis factorial y una vez realizado, en ambos, se obtienen 10 factores que explican un $63.24 \%$ de la varianza total en las universitarias y un $61.25 \%$ en los universitarios. Pese a esta estructura encontrada de 10 factores o constructos, se ha tomado como referentes a interpretar a los 5 primeros factores asumiendo la interpretación intuitiva que marca la gráfica Scree test-gráfico o perfil que en el programa estadístico SPSS versión 20.0 aparece con la opción de gráfico de sedimentación- (Costello y Osborne, 2005). Hemos de ser conscientes de la subjetividad que supone esta elección, pues no se toma como criterio de selección del número de factores los valores absolutos de las varianzas (eigenvalues), pero de nuevo nos guiamos por la pretensión de obtener una estructura lo más clara posible a nivel conceptual.

Por tanto, podemos decir que la percepción o preferencias sobre el empleo o la empresa en la que les gustaría trabajar a este grupo de jóvenes, pueden medirse mediante elementos que se sintetizan, tanto en universitarias como en universitarios, en 5 factores -ver gráficos de 1 a 5-. De esta forma, nos encontramos con que las preferencias de las universitarias y de los universitarios se encuentran determinadas por distintos elementos agrupados en los siguientes factores:

- Factor 1. BUEN CLIMA (universitarias): buscan una empresa donde se pueden sentir bien tratadas, que tenga un buen ambiente, con un trabajo interesante, que intente ajustarse a las capacidades y habilidades de las personas empleadas y que les permita seguir formándose. Una empresa que aporta satisfacción profesional y sentimiento de utilidad personal por lo realizado. Una empresa que permite a las personas desarrollar toda su vida profesional -ver gráfico 1-.

- Factor 1. CREATIVIDAD E INNOVACIÓN (universitarios): buscan empresas que promueven y permiten la creatividad, la innovación, la autonomía y que permita asumir retos para aprender. Una empresa que les aporte sentimiento de utilidad - ver gráfico 1-.

- Factor 2. CREATIVIDAD E INNOVACIÓN (universitarias): señalan a una empresa innovadora, creativa, que ofrezca constantes retos y nuevas experiencias para mantenerse continuamente con cambios -ver gráfico 2-.

- Factor 2. TRABAJO EN EQUIPO (universitarios): se decantan por empresas que fomentan la buena relación e integración entre los y las trabajadoras y en las que se 
pueda orientar y dar servicio, siendo además dicho servicio de utilidad para la sociedad -ver gráfico 2-.

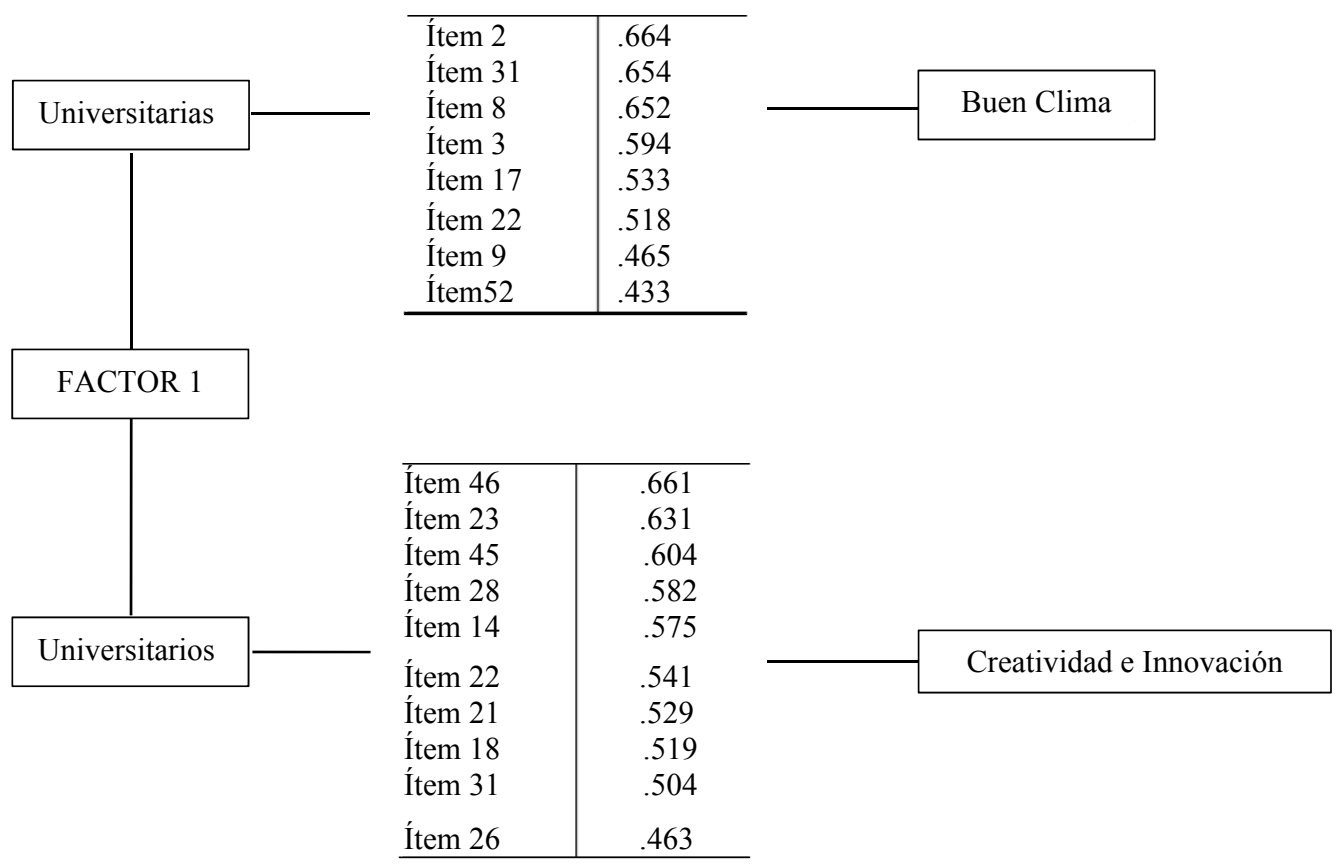

Gráfico 1. Elementos y valores que saturan en Factor 1

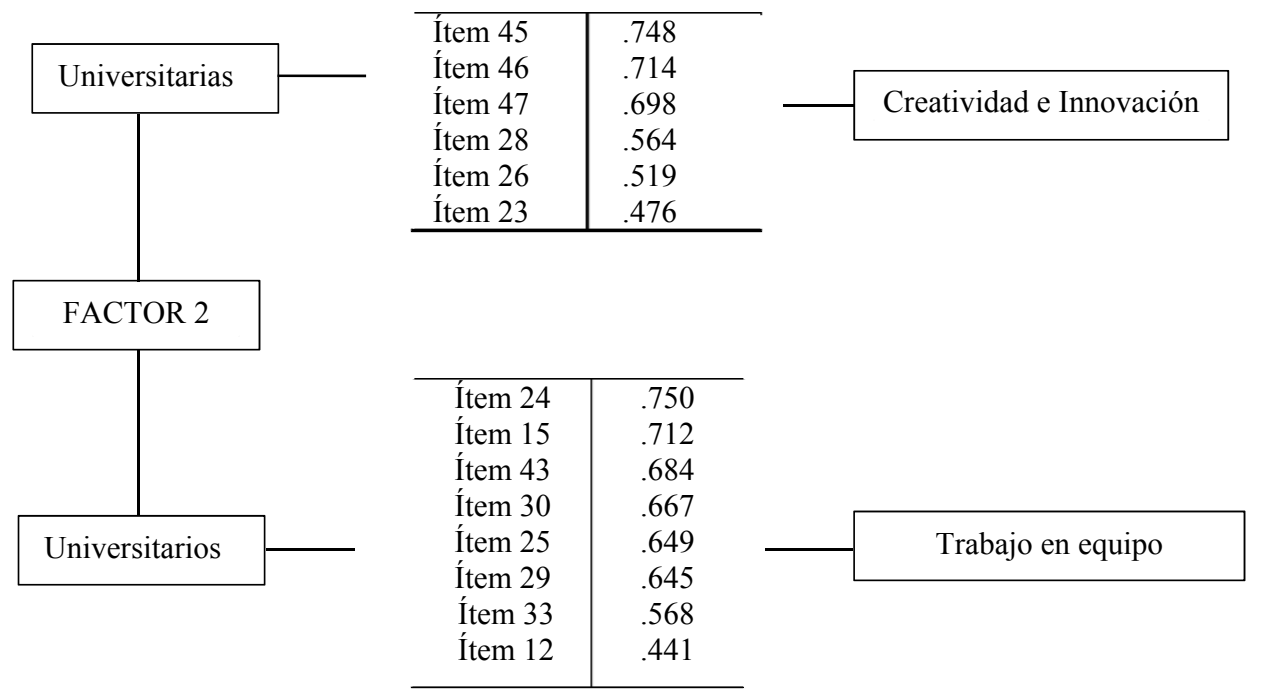

Gráfico 2. Elementos y valores que saturan en Factor 2 
- Factor 3. TRABAJO EN EQUIPO (universitarias): señalan una empresa que potencia los rasgos sociales como el trabajo en equipo, la aceptación de compañeros/as y que fomenta el mantener las buenas relaciones -ver gráfico 3-.

- Factor 3. ÉXITO (universitarios): buscan una empresa que aporte prestigio y éxito profesional, con posibilidad directiva y donde puedan ser admirados y recompensados con promoción laboral. Es importante la capacidad para generar autoempleo y la posibilidad de crear su propia empresa -ver gráfico 3-.

- Factor 4. ÉXITO (universitarias): apuestan por una empresa que aporte al trabajador/a reconocimiento, éxito y prestigio profesional. Es importante la capacidad para el desarrollo de autoempleo y la posibilidad de llegar a crear una empresa -ver gráfico 4-.

- Factor 4. BIENESTAR LABORAL Y PERSONAL (universitarios): valoran las empresas que tienen un buen ambiente y que desarrollan trabajos interesantes. Además, una empresa en la que se establezcan estrategias que permitan la conciliación con la vida familiar -ver gráfico 4-.

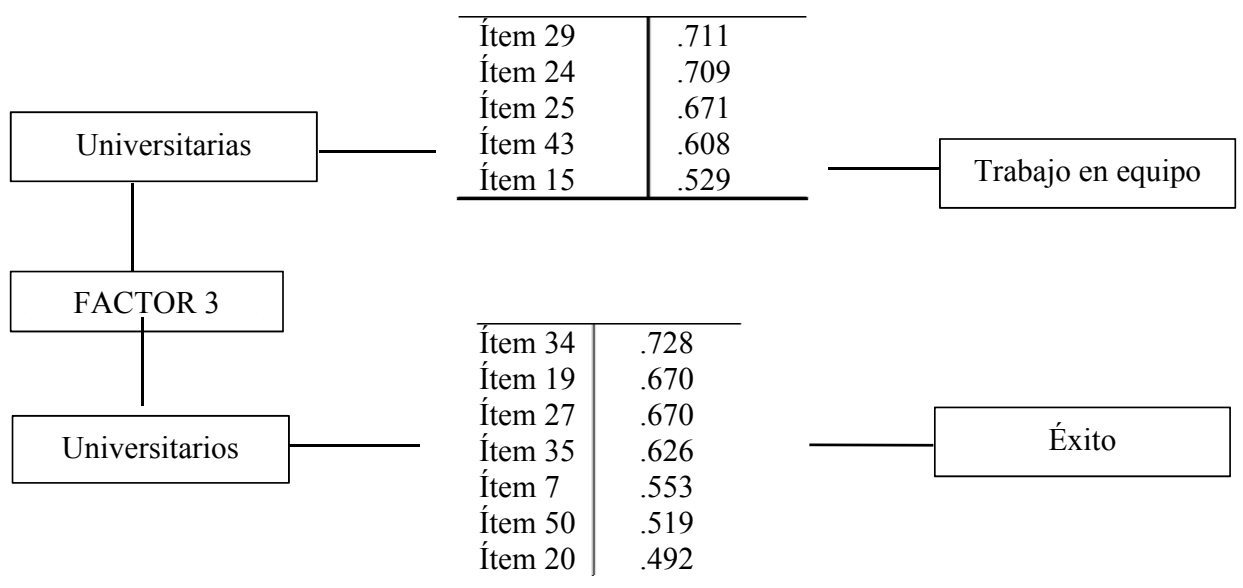

Gráfico 3.Elementos y valores que saturan en Factor 3

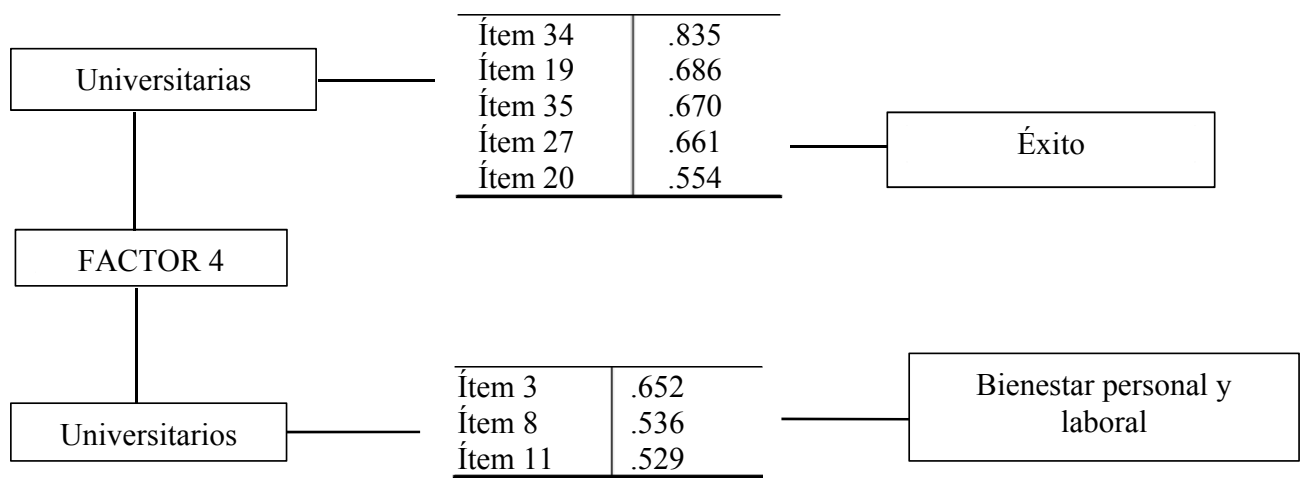

Gráfico 4.Elementos y valores que saturan en Factor 4 
- Factor 5. BUENOS INGRESOS (universitarias): prefieren una empresa que aporte buena remuneración económica puesto que los buenos ingresos son más importantes que el prestigio, la seguridad laboral o la propia satisfacción -ver gráfico 5-.

- Factor 5. BUENOS INGRESOS (universitarios): buscan una empresa que aporte buena remuneración económica puesto que los altos ingresos son más importantes que el prestigio, la seguridad laboral o la propia satisfacción profesional - ver gráfico 5-.

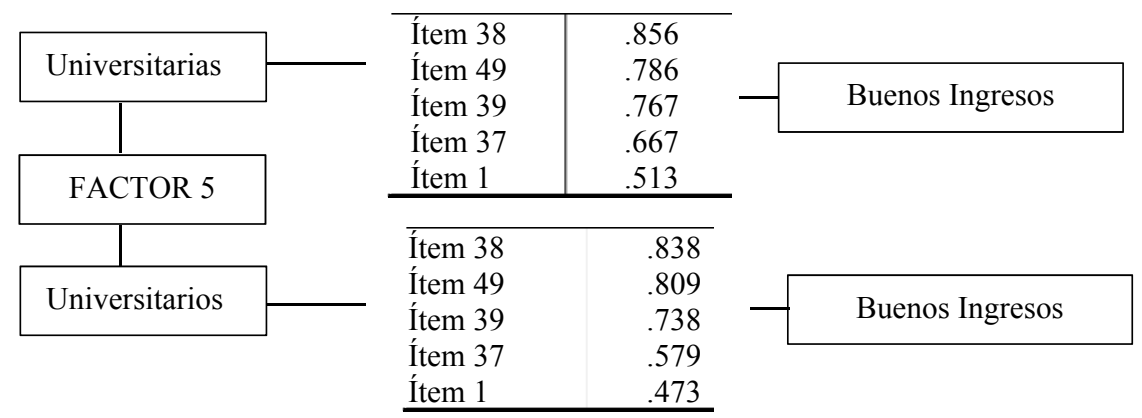

Gráfico 5. Elementos y valores que saturan en Factor 5

Con esta revisión, comprobamos que se obtiene una estructura factorial similar, entre universitarias y universitarios en cuanto a los elementos que se encuentran agrupados en los factores, pero diferenciada en cuanto a la agrupación y orden de los factores o constructos que la componen -ver tabla 5-:

\begin{tabular}{ccc}
\hline Factores & $\begin{array}{c}\text { Estructura factorial } \\
\text { Universitarias }\end{array}$ & $\begin{array}{c}\text { Estructura factorial } \\
\text { Universitarios }\end{array}$ \\
\hline FACTOR I & Buen Clima & Creatividad e Innovación \\
FACTOR II & Creatividad e Innovación & Trabajo en equipo \\
FACTOR III & Trabajo en equipo & Éxito \\
FACTOR IV & Éxito & Bienestar laboral y profesional \\
FACTOR V & Buenos Ingresos & Buenos Ingresos \\
\hline
\end{tabular}

Tabla 5. Ordenación de factores

En síntesis, a partir de estos resultados de la estructura factorial planteada según el género y tomando el orden y el peso de los elementos como un indicador de prioridad, señalamos que para las mujeres universitarias lo que más explica sus preferencias son elementos tales como: que el trabajo les haga sentirse bien tratadas (ítem 2), que sean trabajos interesantes y gratificantes (ítem 8), con un buen ambiente de trabajo (ítem 3), ajustado a sus competencias (ítem 17) y que les permita seguir formándose (ítems 9 y 10). A ellas, el trabajo les debe aportar el sentimiento de que hacen algo útil (ítem 22) y por último señalan que la empresa en la que desearían trabajar es aquella que les permita desarrollar toda su vida profesional (ítem 52). 
Mientras que para los universitarios, lo que más explica sus preferencias viene marcado por aquellas empresas que les permiten tener iniciativas propias (ítem 14) y autonomía en la toma de decisiones (ítem 18). Un trabajo que les aporte nuevas experiencias (ítem 23), innovación (ítem 28), sentimiento de que hacen algo útil (ítem 22), aprendizajes constantes (ítem 21), satisfacción por aquello que realizan (ítem 31) y retos constantes (ítem 26). A la hora de señalar la empresa en la que desearían trabajar destacan como máxima preferencia trabajar en una empresa en la que puedan ser creativos e innovadores (ítems 46 y 45).

Por último y dentro de la estructura factorial de ambos grupos, también nos podemos encontrar con elementos que si bien aparecen en la estructura de factores del grupo de las universitarias no lo hacen en los universitarios y viceversa. En este sentido, se muestra que las universitarias prefieren algunas características en la futura empresa o en su trabajo que no son señaladas por los universitarios. Así, valoran ser bien tratadas (ítem 2); que su tarea se ajuste a sus competencias (ítem 17) y poder seguir formándose en su profesión (ítem 9) llegando incluso, a desarrollar toda su vida profesional en la misma empresa (ítem 52).Y los universitarios, prefieren otras, como que les permita conciliar el trabajo con su vida familiar (ítem 11); que les permita tener iniciativas (ítem 14); ser autónomos en la toma de decisiones (ítem 18) y que sea útil para la sociedad (ítem 12). Además, desearían trabajar en una empresa en la que la tarea a realizar les aporte aprendizajes constantes (ítem 21); de servicio y orientación a otras personas (ítems 30 y 33) y donde puedan dirigir y no ser dirigidos (ítem 50).

\section{Conclusión}

Atendiendo a los resultados encontrados tras los análisis desarrollados, creemos relevante destacar que existen diferencias entre las percepciones que las universitarias y los universitarios tienen respecto a la empresa en la que les gustaría trabajar al terminar su periodo de formación en la universidad en un $65.38 \%$ de las cuestiones planteadas. De lo cual, podríamos plantear que se siguen encontrando diferencias sesgadas por la identidad de rol y el estereotipo de género de los y las estudiantes, en las aulas universitarias.

Específicamente en este estudio se constata que, al decidirse por un trabajo, los universitarios consideran más importante que sus compañeras elementos tales como que éste sea prestigioso -aunque no por encima de tener buenos ingresos- $\mathrm{y}$ el poder llegar a crear su propia empresa, siendo su empresa ideal aquella en la que pueda dirigir a los demás y no ser dirigido. Mientras, las universitarias dan mayor importancia al buen trato, buen clima, a la sensación de utilidad, de reconocimiento entre sus compañeros/as y/o responsables de la empresa. Es decir, las universitarias manifiestan una mayor necesidad de reconocimiento social que los universitarios. Visión que coincide con los resultados hallados en el estudio de Ventura y Quero (2013: 141) donde se destaca que para las mujeres es menos relevante poder llegar a crear una empresa que para los hombres o en el de Sánchez et al., (2011) en el que se concluye que sí que existen diferencias de género, al conceder ellas más importancia al desarrollo personal y en aspectos que contribuyen a establecer relaciones personales, 
autorrealización profesional (actualización, iniciativa, innovación, formación...), así como que ellos mantienen motivaciones más delimitadas y orientadas a lo instrumental.

Sin embargo, y pese a estos aspectos comentados, debemos también resaltar que tras el análisis factorial realizado en nuestro estudio, se aprecia un cambio de tendencia en cuanto a las preferencias que son marcadas por los y las jóvenes ante el trabajo. Aunque el ingreso económico y la seguridad del puesto laboral sigue siendo relevante, también se aprecia una clara tendencia a preferencias por trabajos interesantes y gratificantes, en definitiva, centrados en los procesos de autorrealización personal. Cambios que aparecen quizá más radicales en los universitarios quienes señalan preferencias que hasta ahora no marcaban como son el valorar que su trabajo les permita la conciliación familiar o el desarrollar trabajos que suelen considerarse más feminizados (como dar servicio a otras personas); aunque también en las universitarias se producen cambios hacia la asunción de retos constantes o desarrollar nuevas experiencias.

Tras estos hallazgos, podemos comprobar los cambios que ya son patentes entre los estudiantes universitarios que trabajan en la línea de concepciones más actuales de lo masculino. Donde, se conciben y aprecian aspectos como la corresponsabilidad entre hombres y mujeres en el trabajo doméstico, cuidado de personas dependientes o en otros aspectos; la reorganización y reparto del tiempo que las mujeres y los hombres dedican al proceso productivo y al reproductivo. Promoviendo el desarrollo de un nuevo modelo de relacionales laborales y empleo de calidad que facilite la corresponsabilidad para la conciliación de la vida familiar y laboral, y rompiendo con esquemas tradicionales donde los hombres percibían el empleo como una obligación, donde se hallaban las actividades derivadas de su papel social preeminente, con dedicación exclusiva al desarrollo profesional y absorbiendo su disponibilidad total del tiempo, entre otros. En cuanto a la interpretación de los constructos o factores que subyacen en las agrupaciones de los elementos que hemos obtenido, tras el análisis de componentes principales, podemos señalar que:

- Hay una coincidencia en ambos géneros de lo que supone tener buenos ingresos.

- En ambos la creatividad se manifiesta en una empresa innovadora con retos constantes y que ofrece nuevas experiencias, pero además, los universitarios añaden otras características a creatividad como es permitirles tener iniciativa, aprendizaje constante y autonomía en las decisiones.

- En lo referente al trabajo en equipo, si bien ambos grupos entienden que es potenciar rasgos sociales, aceptación de compañeros/as y fomentar buena relación, los universitarios añaden que un trabajo en equipo es el que puede orientar y dar servicio a otros/as, siendo además dicho servicio de utilidad para la sociedad.

- El éxito es entendido como conseguir reconocimiento, prestigio profesional, capacidad para el desarrollo de autoempleo y la posibilidad de llegar a crear una empresa. Pero además, para los universitarios supone el poder promocionar y el dirigir en lugar de ser dirigidos.

- Una percepción diferente en lo se comprende por el clima laboral, dado que si los universitarios lo que desean en fundamentalmente sentirse bien porque les gusta un 
buen ambiente y que se les facilite la conciliación con su vida laboral, las universitarias entienden por ello empresas en las que se les trate bien, se sientan satisfechas, su trabajo se ajuste a lo que saben hacer y que les permita una mayor estabilidad laboral/profesional.

Así pues, nos parece relevante destacar que todos estos factores subyacen de forma sustancial a la nueva concepción del trabajo y el empleo, donde la creatividad, el trabajo en equipo y el clima son elementos muy relevantes en la línea de las nuevas exigencias empresariales al mismo tiempo que el éxito y los ingresos.

Sin embargo, no queremos concluir este trabajo sin remarcar que, en líneas generales, los resultados aquí obtenidos nos muestran que los y las estudiantes universitarios/as, continúan de forma general y a día de hoy desarrollando sus preferencias laborales sesgadas por estereotipos de género. Es necesario que el profesorado de los distintos niveles educativos asumamos el reto de la coeducación realizando acciones de intervención desde el ámbito de la orientación profesional en las que se busquen romper con estas tendencias y preferencias sesgadas. Así pues, la educación universitaria actual refuerza las disparidades de intereses entre los géneros y no contribuye al aprendizaje de la igualdad, aunque ésta sea valorada por una gran cantidad de docentes y personas responsables del mundo educativo.

\section{Referencias bibliográficas}

BARBERÁ, E.; RAMOS, A. Y CANDELA, C. (2006). Mercado laboral y estereotipos de género. Revista de Psicología Social Aplicada, 16, (1-2), 165-179.

BLOSCH, D. P. (2005). Complexity, chaos and nonlinear dynamics: a new perspective on career development theory. The career development quarterly, 53 (3), 194-207.

CASTILLO, G. y RODRÍGUEZ SEDANO, A. (2011). De la Universidad al puesto de trabajo. Estrategias y recursos para acceder al primer empleo. Madrid: Ediciones Pirámide.

De ARMOND, S.; TYE, M.; CHEN, Y.P.; KRAUS, A.; ROGERS, A. y SINTEK, E. (2006). Age and gender stereotypes: New challenges in a changing workplace and workforce. Journal of Applied Social Psychology, 36, (9), 2184-2214.

ELEJABEITA, C. (1995). Las pulsiones sociales de la variable sexo en la elección de las carreras. Pioneras y marginados. Madrid: Ministerio de Educación y Ciencia.

EUROPEAN VALUES STUDY AND GESIS DATA ARCHIVE FOR THE SOCIAL SCIENCES (2008). European Values Study Master Questionnaire en EVS 2008 Method Report Country Report - Spain.Luxembourg: GESIS.

FALKNER, W. (2000). The power and the pleasure? A research agenda for making gender stick to engineers. Science, Technology and Human Values, 25 (1), 87-119.

FORSON, C. y ÖZBILGIN, M. (2003). Dot-com women entrepreneurs in the UK. Entrepreneurship and Innovation. February. 13-24. 
FUNDACIÓN UNIVERSIDAD-EMPRESA (2009). Informe de resultados. Compromiso en la empresa. Compromiso laboral de los universitarios. Diagnóstico comparativo 2008/2009. Madrid: Fundación Universidad-Empresa. Recuperado de www.fue.es/HTML/IMAGES/50545212/91151064634.pdㅍ

GÓMEZ, C. (2001). Mujeres y trabajo: principales ejes de análisis. Papers, 63/64, 123 140.

GONZÁLEZ O. (2001). Actitudes hacia el trabajo y la creación de empresas según género. Boletín Económico del ICE, 2709.

IGLESIAS, C. y Llorente, R. (2010). Evolución reciente de la segregación laboral por género en España. Revista universitaria de Ciencias del Trabajo, 11, 81-105.

INGLEHART, R. (1998). Modernización y posmodernización. El cambio cultural, económico y político en 43 sociedades. Madrid: CIS.

KULIS, S.; SICOTTE, D. y Collins, S. (2002). More than a pipeline problem: Labor supply constraints and gender stratification across academic science disciplines. Research in Higher Education, 43 (6), 657-691.

MARTÍN, J. y MIGUEL, A. de (1982). Sociología de las profesiones. Madrid: Centro de Investigaciones Sociológicas.

NASH, M. y MARRE, D. (2003). El desafío de la diferencia: representaciones culturales e identidades de género, raza y clase. Bilbao: Universidad del País Vasco.

NAVARRO, C. y CARERO, A. (2012). Análisis de las diferencias de género en la elección de estudios universitarios. Estudios Sobre Educación: ESE. 22, 115-132.

NAVARRO, C. y SALLÉ, M. A. (Coords.) (2007). Diagnóstico y autodiagnóstico de barreras de género. Posicionamiento de la mujer ante el empleo. Sevilla: Colegio Oficial de Psicología de Andalucía Occidental.

OLIVARES, Ma . C. y Olivares C. (2013). Impacto de los estereotipos de género en la construcción de la identidad profesional de estudiantes universitarios. REOP. 24 (1), $121-131$.

PADILLA, Ma. T. (2002). Desarrollo profesional femenino: la identidad de la mujer en el mundo laboral. En E. Moreno y S. Villegas (Eds.). Introducción a los estudios de la mujer. Una mirada desde las Ciencias Sociales (pp.73-95) Huelva: Diputación Provincial. Seminario de Estudios de la Mujer. Universidad de Huelva.

PATTON, W. y MCMAHON, M. (2006). Career development and systems theory. Connecting theory and practice. The Netherlands: Sense Publishers.

PÉREZ-CARBONELL, A. y RAMOS, G. (2013). Expectativas de los estudiantes universitarios de distintas ramas de conocimiento sobre el mundo laboral. En J. Gacel-Ávila y N. Orellana Alonso (Coords.). Educación superior, gestión, innovación e internacionalización (pp. 215-240). Valencia: Universitat de València y Universidad de Guadalajara.PONS, O.; CALVET, M ${ }^{\mathrm{a}}$.D.; TURA SOLVAS, M. y 
Muñoz, C. (2013). Análisis de la Igualdad de Oportunidades de Género en la Ciencia y la Tecnología: Las carreras profesionales de las mujeres científicas y tecnólogas. IC, 9 (1), 65-90.

RODRÍGUEZ, M. J. y SANTOS, F. J. (2008). La actividad emprendedora de las mujeres y el proceso de creación de empresas. Información Comercial Española, 841.

ROSSER, S.V. (1998). Applying feminist theories to women in science programs. Signs. Journal of Women in Culture and Society, 24 (1), 171-201.

RUSSELL, G. y HAWANG, C.P. (2004). The impact of workplace practices on father involvement, en Michael $\mathrm{E}$. Lamb (Ed.) The Role of the father in child development, $4^{\text {th }}$ ed., Hobokem, New jersey, John Wiley \& Sons.

SÁNCHEZ, M.; SUÁREZ, M.; MANZANO, N.; OLIVEROS, L.; LOZANO, S.; FERNÁNDEZ, B. y MALIK, B. (2011). Estereotipos de género y valores sobre el trabajo entre los estudiantes españoles. Revista de Educación, 355, 331-354.

SEBASTIÁN, A.;SÁNCHEZ, M. F.; SUTIL, M. I.; BORONAT, J.; CADIERNO, D. y SOLANO, L.O. (2005). La presencia de estereotipos de género en el sistema educativo como determinante del desarrollo personal y profesional (Estudio descriptivo). Madrid: Dyckinson.

SILVÁN-FERRERO, M. P.; BUSTILLOS, A. y FERNÁNDEZ, M. J. (2005). Género y orientación vocacional. Comunicación presentada en el II Congreso HispanoPortugués de Psicología. Santiago de Compostela.

SUÁREZ, M. (2004). Las mujeres adultas y los procesos de re-inserción laboral. Un estudio en contextos locales de la provincia de Sevilla. Sevilla: Excma. Diputación de Sevilla.

VENTURA, R. y QUERO, Ma . J. (2013). Factores explicativos de la intención de emprender en la mujer. Aspectos diferenciales en la población universitaria según la variable género. Cuadernos de Gestión, 13(1), 127-149.

WAJCMAN, J. (2000). Feminism confronts technology. Cambridge: Polity Press.

\section{Correspondencia con las autoras}

Amparo PÉREZ-CARBONELL

Dpto. MIDE- Facultad de Filosofía y CC. de la Educación

Avd. Blasco Ibañez, 30. 46010 Valencia

e-mail: amparo.perez@uv.es

Genoveva RAMOS SANTANA

Dpto. MIDE- Facultad de Filosofía y CC. de la Educación

Avd. Blasco Ibañez, 30. 46010 Valencia

e-mail: genoveva.ramos@uv.es 